\title{
FES-based control of knee joint to reduce stance phase asymmetry in post-stroke gait: feasibility study
}

\author{
B. Sijobert, C. Fattal, J. Pontier, C. Azevedo Coste
}

\begin{abstract}
In this study, a novel approach is proposed to improve stance phase support symmetry recovery in acute post-stroke individuals, based on knee joint control. Functional electrical stimulation (FES) is delivered to the quadriceps and hamstrings muscles of the paretic limb based on the online estimation of knee angle and support phase. This paper is a proof-of-concept article, which introduces the global approach and some preliminary results obtained on two participants with post-stroke hemiplegia.
\end{abstract}

\section{INTRODUCTION}

$\mathrm{T}_{\mathrm{p}}$ HE process of gait recovery in patients with severe post-stroke hemiplegia requires a significant investment and effort for both the patient and the therapist while not always leading to a safe and autonomous gait. Increasing the chances for the patients of regaining a functional walking gait within limited timeframes represents a major challenge, additionally encouraged by today's trend to reduce as much as possible the length of stay in rehabilitation centers. Several studies have focused their attention on the neurological foot in the chronic phase because a poorly controlled foot dorsiflexion is a factor limiting gait recovery and increasing fall risk. Functional Electrical Stimulation (FES) can be used as an alternative to an orthosis to restore walking by activating paralyzed muscles. FES has been extensively studied to correct drop foot by activating foot dorsiflexors [1] or inducing a withdrawal reflex [2].

FES of the dorsiflexors does not improve all gait deficits associated with hemiplegic gait. Few studies have considered knee joint. However it also plays a major role over the entire gait cycle and especially regarding the support phase quality. In the initial period after stroke, ensuring a safe knee control remains difficult, partly due to associated disorders including a lack of proprioceptive feedback. Classical observed disorders in this population are knee hyperextension during the stance phase (genu recurvatum) and flexed knees (crouch gait). Fixed orthoses are usually used to prevent this by limiting knee flexion or extension over the gait. FES represents also an alternative to produce appropriately timed knee flexion or extension. Bioness L300 Plus@ device proposes a thigh cuff embedding

This work was supported by a funding support (\#2015-2-41) from LabEx NUMEV (Montpellier, France)

B. Sijobert and C. Azevedo Coste are with INRIA, University of Montpellier, France (benoit.sijobert@inria.fr).

C. Fattal and J. Pontier are with CRF La Châtaigneraie, Menucourt, France electrodes stimulating the quadriceps muscles to extend knee at a predefined time during gait based on shank angle observation. Previous studies have investigated the contribution of quadriceps and gluteus maximus stimulation in improving standing balance and weight transfer [3] or hamstrings stimulation associated to foot dorsiflexors stimulation in improving gait performance [4].

In the present work, we aim at ensuring a safe knee joint to encourage patients to rely on their paretic leg and transfer their weight onto it while walking. Depending on the gait phase, the knee extension and flexion are restricted to a safe and physiological range of motion by the adaptive delivery of quadriceps and hamstring electrical stimulation. A sensor network detects the gait phases and knee angle evolution from which stimulation levels are modulated.

\section{MAterial AND Methods}

The protocol presented here has been approved by a national ethical committee and participants have signed an informed consent. Two participants have been included at this stage of the study. The subjects were equipped with 2 inertial measurement units (IMU Bosch@ BNO055) wired to a Raspberry Pi3@. Each IMU embedded a high speed ARM Cortex-M0 based processor and a Kalman Filter directly providing quaternion estimation needed to compute knee angles [5] at a $100 \mathrm{~Hz}$ sampling rate. Two wireless Bluetooth pressure insoles (FeetMe $($, France) were connected to the Raspberry. Stimulation was delivered via a wireless stimulator (Phenix Neo@) to the quadriceps and hamstrings via surface electrodes (Fig 1.).

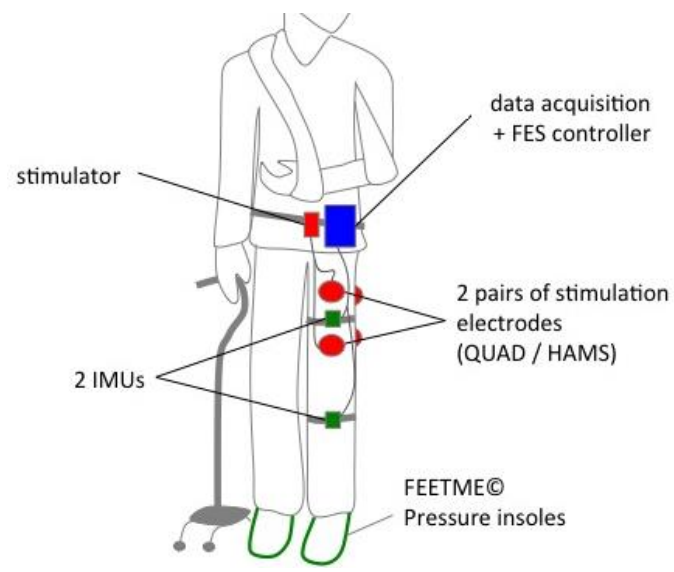

Fig. 1. Setup description: participants were equipped with 2 magnetoinertial sensors wired to a Raspberry located around the waist. 2 foot pressure insoles were inserted in the shoes. FES was delivered via a wireless stimulator to the quadriceps and hamstrings. 
Data from IMUs and pressure insoles were recorded and processed online on the controller embedded in the Raspberry (Fig. 2). An ad-hoc wireless network emulated by the controller enabled to wirelessly access to the algorithm and stimulation parameters so as to emergency stop commands from a PC located in a nearby distance of the subject.

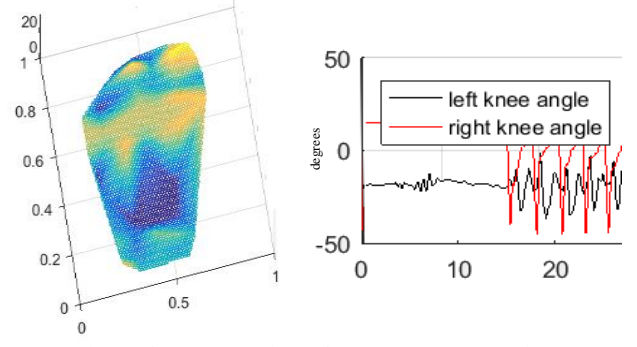

Fig. 2. Illustration of data acquired from the pressure insoles and knee angles estimated from the IMUs.

Insoles were used to online analyze paretic foot support (PFS) in order to discriminate between stance and swing phases. Stimulation could also be delivered just before initial contact (IC) at the end of the swing phase, in order to anticipate a possible genu recurvatum or crouch gait in stance phase and compensate muscular activation latency. When required and depending on the participant's gait pattern, this "pre-stance" stimulation could be triggered either via an online detection of peak knee flexion or when the sagittal angular speed recorded via the gyroscope crossed zero. In stance phase, stimulation was triggered either to quadriceps or hamstrings, depending on the paretic knee angle (PKA) estimation relatively to the knee angle setpoint (KAS) defined by the practitioner as the optimal flexion during stance phase (around $5^{\circ}$ ). A proportional (P) controller adjusted the pulse width depending on the error $\varepsilon$ between PKA and KAS (Fig.3).

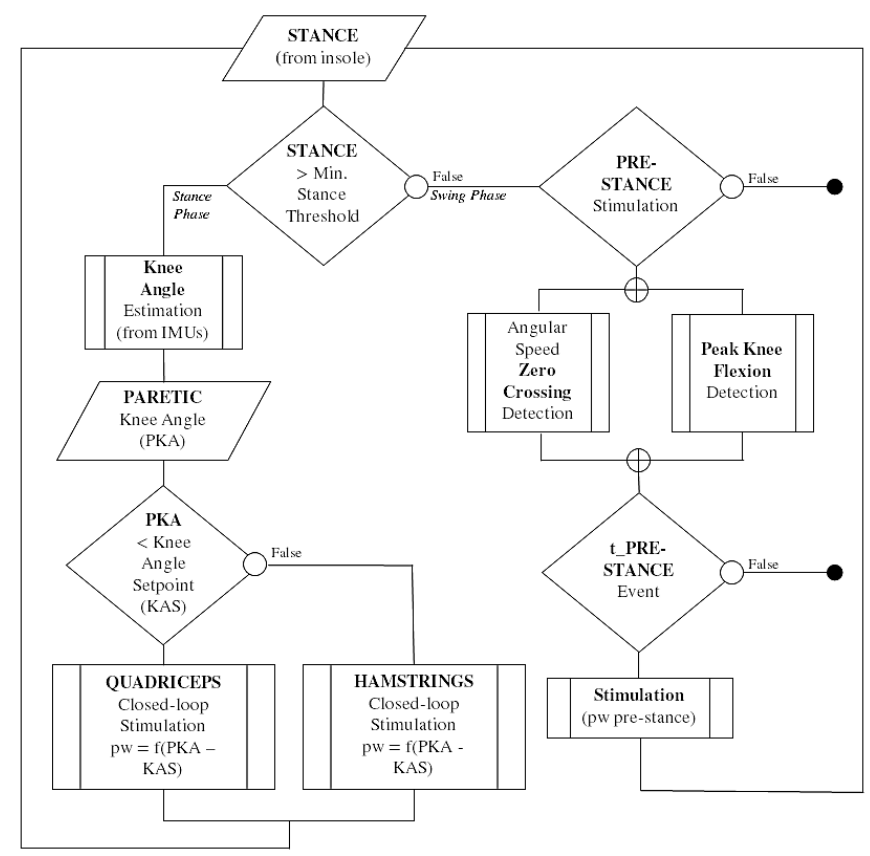

Fig. 3. Flowchart of the decisional algorithm applied online during gait.
Equipped with their usual technical aids (cane, AFO...) participants were asked to perform a $10 \mathrm{~m}$-path walking at a self-selected pace. An oral instruction was given at the beginning of each trial to encourage the participants to transfer their weight onto the paretic leg.

\section{RESULTS}

In both participants the stimulation of quadriceps and hamstrings was successfully delivered accordingly to the decisional algorithm (Fig 4).

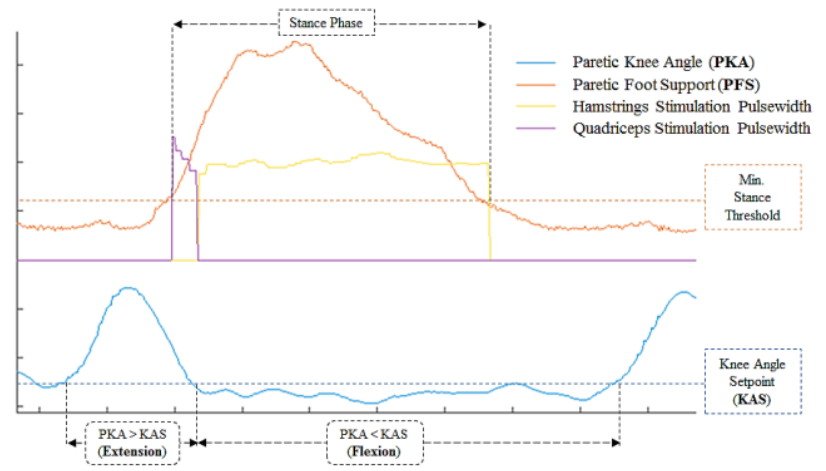

Fig. 4. Example of recorded data during one gait cycle. During stance phase, defined when PFS data (orange) goes above a preset threshold, stimulation of hamstrings (purple) is delivered when PKA is higher than KAS and stimulation of quadriceps (yellow) delivered when PKA lower than KAS. Stimulation pulsewidth is adjusted via a proportional controller depending on the error between PKA and KAS.

\section{CONCLUSION}

Two sessions of three trials were carried out to assess the feasibility of online controlling the stimulation of quadriceps and hamstrings depending on gait phase and observed knee joint angle. Based on foot insoles and inertial sensors the level and timing of the stimulation was adapted to offer a safe knee support. In two patients, the feasibility of our approach was suggested to be promising. In the future, other participants will be included and gait improvements quantified over multiple indicators.

\section{REFERENCES}

1. W. T. Liberson, M. E. Holmquest, D. Scott, M. Dow. 'Functional electrotherapy: stimulation of the peroneal nerve, synchronized with the swing phase of gait of hemi-paraplegic patients' in Arch. Phys. Med., 1961, 42, 101-105.

2. E. Spaich, N. Svaneborg, H.R. Jørgensen, O.K. Andersen, 'Rehabilitation of the hemiparetic gait by nociceptive withdrawal reflex-based functional electrical therapy: a randomized, singleblinded study' in J Neuroeng Rehabil., 2014, 11:81.

3. D. Kunkel, R.M. Pickering, M. Burnett, J. Littlewood, J.H. Burridge, A. Ashburn, 'Functional Electrical Stimulation With Exercises for Standing Balance and Weight Transfer in Acute Stroke Patients: A Feasibility Randomized Controlled Trial.' in Neuromodulation: Tech. at the Neural Interface, 2013, 16: 168-177.

4. S. Springer, J.-J. Vatine, A. Wolf, Y. Laufer, 'The effects of dualchannel functional electrical stimulation on stance phase sagittal kinematics in patients with hemiparesis.' in Journal of Electromyography and Kinesiology, 2013, 476-482.

5. B. Sijobert, F. Feuvrier, J. Froger, D. Guiraud, C. Azevedo-Coste, 'A Sensor Fusion Approach for Inertial Sensors Based 3D Kinematics and Pathological Gait Assessments: Toward an Adaptive Control of Stimulation in Post-Stroke Subjects', in IEEE Engineering in Medicine and Biology Society conference, 2018. 